\title{
AHP-Based Features Analysis of Automotive Sketches
}

\author{
Qiang Li ${ }^{1, a}$, Changhai Yang, ${ }^{1, b, *}$, Zhao Zhang, ${ }^{1, c}$, Yongjian Gong, ${ }^{2, d}$, and Cuixian Xuan ${ }^{2, e}$ \\ ${ }^{1}$ State Key Laboratory of Automobile Simulation and Control, Jilin University, Jilin, P. R. China \\ ${ }^{2}$ Jinhua Polytechnic, Jinhua, Zhejiang, P. R. China \\ aq1i@jlu.edu.cn, ${ }^{\mathrm{b}}$ ych@jlu.edu.cn, ${ }^{\mathrm{c}}$ zhangzhao@jlu.edu.cn, \\ djhxuancx@qq.com, and ejhjdgyj@163.com \\ *corresponding author
}

Keywords: Automotive styling, Sketching, Stroke, Feature extraction, AHP.

\begin{abstract}
This paper tries to give a mathematic explanation for the classification of the most important features in the automotive sketches. Based on the understanding of $2 \mathrm{D}$ sketches and their roles in automotive styling, this paper has investigated the expressions, styles and features of automotive sketches, and proposed a reasonable classification for the form lines of automotive features, which are the most important to be sketched in the future 3D automotive sketching system. Some typical automotive sketches have been selected and analyzed according to the patterns of single stroke, combined line and surface, and light color. The features have been extracted by the experiment from the four forms: form lines, components, form shadings and non-form shadings. The analytic hierarchy process (AHP) has been applied to deal with the experimental data. It is proved that the classification and the importance analysis of features are reasonable.
\end{abstract}

\section{Introduction}

In the conceptual design stage of automotive styling, two-dimensional sketches are widely adopted because of its freedom, arbitrary, easy to record, and other advantages. Sketches have a variety of forms and styles, some of them are regular single outlines, wild scribbles using colored pencils, expressions with large markers in sharp contrast, or evenly smearing with crayons. Sketch is a tool for stylists' expression of creative design. The forms and styles of sketches are changed with different designers and different factors such as the idea of the concept.

Since the forms and styles of sketches are so rich and varied, how can people identify the information contained in the sketch, which is considered as stylist's important tool to communicate with others and as a basis to create three-dimensional digital model and clay model? In the extraction of the feature lines of car styling, Zhao [1-2] gave their definitions to the feature lines. They are divided the lines in the automotive sketches into the top, modeling and regional ones; the modeling features in the three-dimensional digital model into the main, transitive and additional ones, and accordingly the main, transitive and additional lines as well. Jiang [3] presented the further refined automotive styling features, which are classified into the ones of top surface, left and 
right surfaces, front and rear surfaces. By proposing the concept of "feature set", he put all detailed features of automotive styling in the feature sets, and used the "quasi-three-dimensional" evaluation technique of styling [4] to do the division and extraction. The line is composed of numerous points, but it can also be used to form a plane or surface, and it is a "nexus" styling elements [5]. As an important tool to express the contours and features in the automotive sketches, feature lines are often used by stylists, while various famous automotive brands inherit and keep changing continually their own feature lines in styling. Zhang [6] presented the concept of modeling gene from the point of view of biological form generation and evolution. Zhu [7] had investigated Audi A4 waistline from the perspective of visual psychology. A more important aspect in sketch research is the sketch understanding and recognition techniques. McGown [8] proposed the "complexity" concept by investigating a large number of sketches. The sketches are divided into five generalized complexities, and each is defined. Tovey [9] proposed a classification about automotive conceptual sketches that sketches were divided into four layers: form shading, non-form shading, form lines and components.

\section{Features in Automotive Sketches}

\subsection{Styles of Automotive Sketches}

In this paper the styles in automotive sketches are divided into the following three forms:

1) Single line. Single line is the most common form used in the conceptual design sketches. The tools, such as pencils, pens, or fine points, are simple and easy to use. Single lines are used to express remarkably the main features of automobile, for example, body feature lines, the turning point of surfaces, and general shadows in perspective view, etc. The desired effect can be controlled through the thickness and density of single line (Figure 1a). It is necessary to keep the coherence and consistency of single lines to avoid the casual exaggeration from feeling, or the blind pursuit of hand techniques for the expression [10]. Various stream-line generations among the different algorithms focus mainly on velocity vector interpolation, numerical integration, step selection of integration, seed point arrangement, and termination conditions of stream-lines [5].

2) Combined line and surface. Based on the single line form, this form uses the tools such as wide-tip pen, script liner, marker pen, watercolor brush and so on. When using this form, it should be clear where the surfaces are needed to be expressed [10], such as the turns of surface and dark shadows. This form not only keeps the effect of single line, but also shows the sense of space and level, with a strong artistic charm to make the sketch more varied (Figure 1b).

3) Light color. It is a combination of the above two forms, and decorated with systematic color performance, usually using markers, watercolor brushes or color powders (Figure 1c). At the same time of using the first two forms, the basic principles of color relationships of the scheme expressed should be met in color and brightness in a quick and easy way. It needs not be comprehensive or too much modification. The merit of this form is to be able to express completely the shapes and colors of product, to get more real visual artistic effect [10].
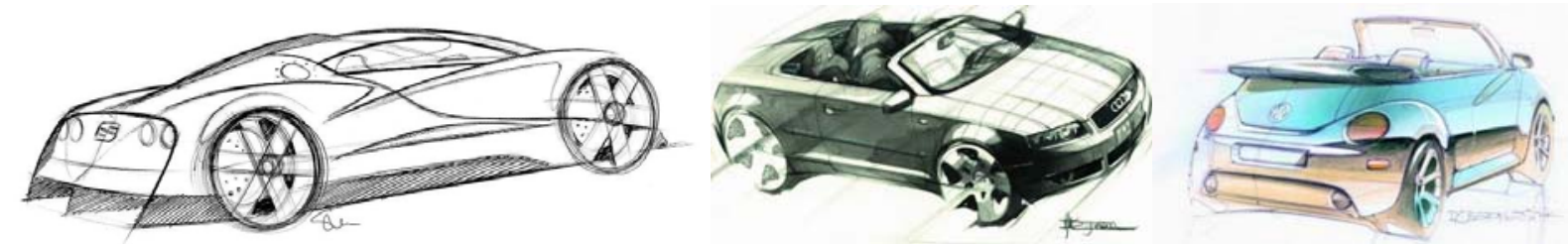
a. Single lines [11]
b. Line and surface combined [12]
c. Light color [10]

Figure 1 Three forms of automotive sketches. 


\subsection{Feature Lines in Styling Elements}

In the automotive conceptual design, the feature line of vehicle is an important tool and means of styling characteristic expression. The feature lines in this paper are divided into three sections: front, side and rear area of autobody (Figure 2). These feature lines in the sketch contain the information both in the connotation of the form and structure, there are specific structural constraints and styling connotation [13].

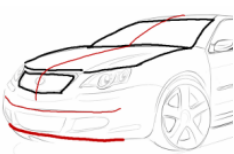

a. Front

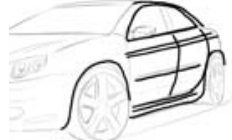

b. Side

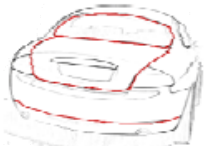

c. Rear

Figure 2 Feature lines of autobody [16-17].

\section{Extraction of Sketch Features}

\subsection{Decomposition Analysis of Sketch}

It is necessary to know which the features are important. The form lines, components, form shading and non-form shading are extracted from the similar style sketches, and then named and defined in Photoshop. Since the sketch of a car in the front 3/4 view and the rear 3/4 view can contain all the three-dimensional information, and the perspective view is significant to free-hand sketching, four sets of perspective views are selected for analysis in each form of sketches, respectively.

One set of sketches in the front 3/4 view and the rear 3/4 view have been used for the single-line form analysis (Figure 3). The form lines, components, form shading and non-form shading are listed at the right of sketch from top to bottom. The form of combined line and surface and the one of light color are shown in Figure 4 and Figure 5, respectively.
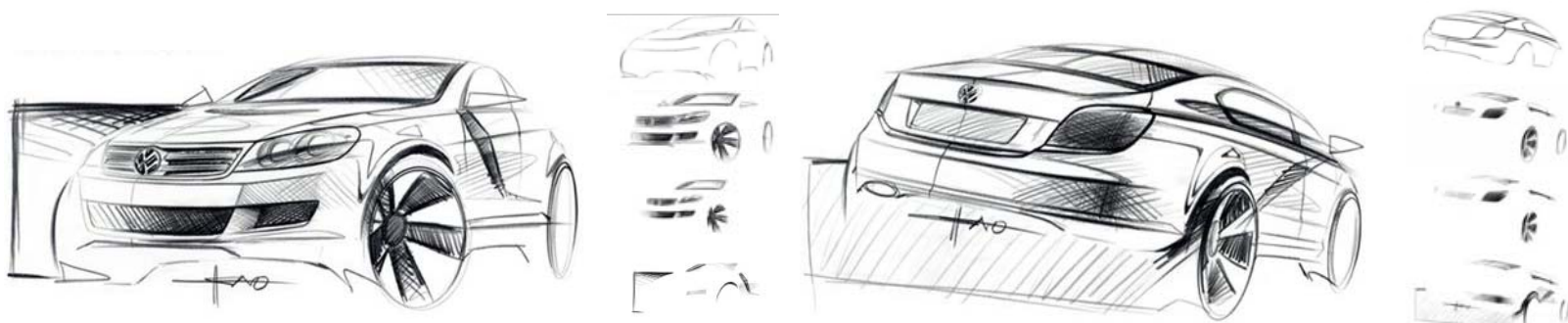

Figure 3 Single line sketches (Left: front 3/4 view [16], Right: rear 3/4 view [17]).
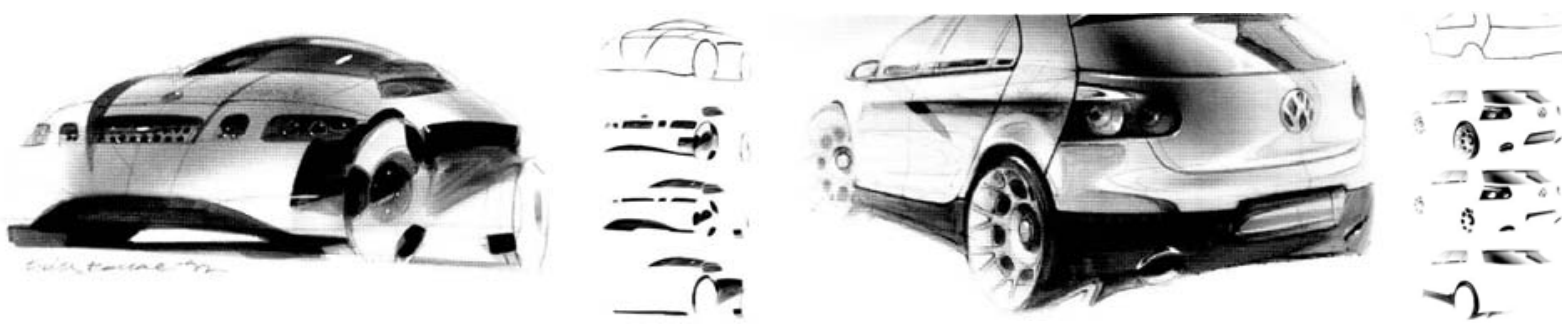

Figure 4 Line and surface combined sketches (Left: front 3/4 view [10], Right: rear 3/4 view [10]). 

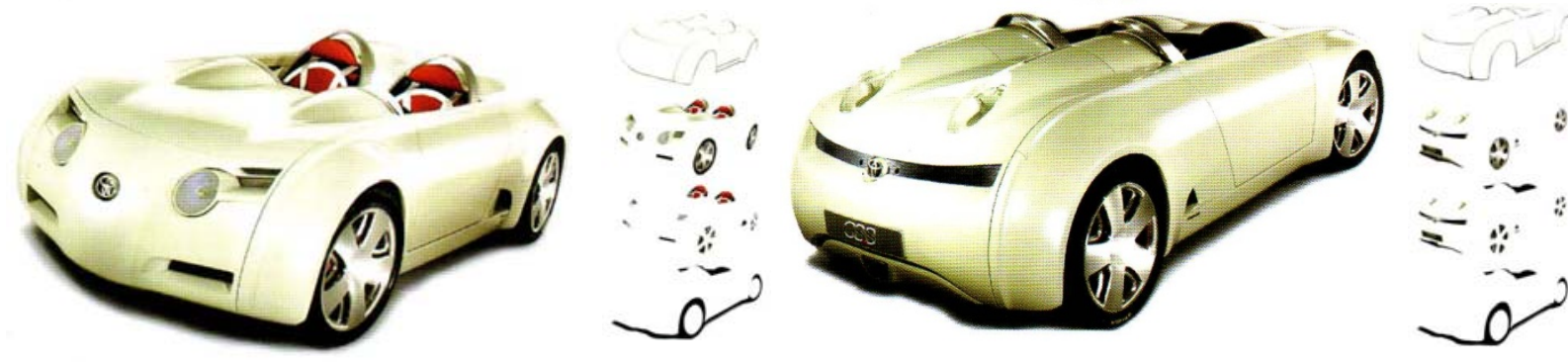

Figure 5 Light color sketches (Left: front 3/4 view [10], Right: rear 3/4 view [10]).

\subsection{Data Processing}

Twelve groups of sketches are analyzed and the frequency of each kind of feature line existing is accounted as well. The samplings of the three forms have been taken in four times, and the frequency of sampling can reflect the level of importance.

1) Form Line. In the sketch, much of the shadow information cannot be used accurately to define the surface boundaries of automobile in most cases, it is necessary to use form lines to provide the form of automobiles (Table 1).

Table 1 Form lines.

\begin{tabular}{|c|l|l|l|l|l|c|c|c|c|c|c|c|c|}
\hline No. & \multicolumn{1}{|c|}{ Features } & \multicolumn{3}{|c|}{ Single Line } & \multicolumn{3}{c|}{ Line \& Surf. } & \multicolumn{3}{c|}{ Light color } \\
\hline 1 & Roof line & $\bullet$ & $\bullet$ & $\bullet$ & $\bullet$ & $\bullet$ & $\bullet$ & $\bullet$ & $\bullet$ & & $\bullet$ & $\bullet$ & $\bullet$ \\
\hline 2 & $\begin{array}{l}\text { Front windscreen } \\
\text { profile }\end{array}$ & $\bullet$ & $\bullet$ & $\bullet$ & $\bullet$ & $\bullet$ & $\bullet$ & $\bullet$ & $\bullet$ & $\bullet$ & $\bullet$ & $\bullet$ & $\bullet$ \\
\hline 3 & Front hood line & $\bullet$ & $\bullet$ & $\bullet$ & $\bullet$ & $\bullet$ & $\bullet$ & $\bullet$ & $\bullet$ & $\bullet$ & $\bullet$ & $\bullet$ & $\bullet$ \\
\hline 4 & Side hood line & $\bullet$ & $\bullet$ & $\bullet$ & $\bullet$ & $\bullet$ & $\bullet$ & $\bullet$ & $\bullet$ & $\bullet$ & $\bullet$ & $\bullet$ & $\bullet$ \\
\hline 5 & Grille line & $\bullet$ & $\bullet$ & $\bullet$ & $\bullet$ & $\bullet$ & $\bullet$ & $\bullet$ & $\bullet$ & $\bullet$ & $\bullet$ & $\bullet$ & $\bullet$ \\
\hline 6 & Front bumper line & $\bullet$ & $\bullet$ & $\bullet$ & $\bullet$ & $\bullet$ & $\bullet$ & $\bullet$ & $\bullet$ & $\bullet$ & $\bullet$ & $\bullet$ & $\bullet$ \\
\hline 7 & Middle line & $\bullet$ & $\bullet$ & $\bullet$ & $\bullet$ & $\bullet$ & $\bullet$ & $\bullet$ & $\bullet$ & & & & \\
\hline 8 & Side window profiles & $\bullet$ & $\bullet$ & $\bullet$ & $\bullet$ & $\bullet$ & $\bullet$ & $\bullet$ & $\bullet$ & $\bullet$ & $\bullet$ & $\bullet$ & $\bullet$ \\
\hline 9 & Waist line & $\bullet$ & $\bullet$ & $\bullet$ & $\bullet$ & $\bullet$ & $\bullet$ & $\bullet$ & $\bullet$ & $\bullet$ & $\bullet$ & $\bullet$ & $\bullet$ \\
\hline 10 & Shoulder line & $\bullet$ & $\bullet$ & $\bullet$ & $\bullet$ & $\bullet$ & $\bullet$ & & $\bullet$ & & $\bullet$ & $\bullet$ & $\bullet$ \\
\hline 11 & Side scuff line & $\bullet$ & $\bullet$ & & & & & & & & & $\bullet$ & \\
\hline 12 & Skirt line & $\bullet$ & $\bullet$ & $\bullet$ & $\bullet$ & $\bullet$ & $\bullet$ & & $\bullet$ & $\bullet$ & & & \\
\hline 13 & Side chassis line & $\bullet$ & $\bullet$ & $\bullet$ & $\bullet$ & $\bullet$ & $\bullet$ & & $\bullet$ & $\bullet$ & $\bullet$ & $\bullet$ & $\bullet$ \\
\hline 14 & Wheel cover line & $\bullet$ & $\bullet$ & $\bullet$ & $\bullet$ & $\bullet$ & $\bullet$ & $\bullet$ & $\bullet$ & $\bullet$ & $\bullet$ & $\bullet$ & $\bullet$ \\
\hline 15 & Door profile & $\bullet$ & $\bullet$ & $\bullet$ & $\bullet$ & $\bullet$ & $\bullet$ & & $\bullet$ & $\bullet$ & $\bullet$ & $\bullet$ & $\bullet$ \\
\hline 16 & Rear window profile & $\bullet$ & $\bullet$ & $\bullet$ & $\bullet$ & $\bullet$ & $\bullet$ & $\bullet$ & $\bullet$ & $\bullet$ & $\bullet$ & $\bullet$ & $\bullet$ \\
\hline 17 & Front trunk lid line & $\bullet$ & $\bullet$ & $\bullet$ & $\bullet$ & $\bullet$ & $\bullet$ & $\bullet$ & $\bullet$ & $\bullet$ & $\bullet$ & $\bullet$ & $\bullet$ \\
\hline 18 & Side trunk lid line & $\bullet$ & $\bullet$ & $\bullet$ & $\bullet$ & $\bullet$ & $\bullet$ & $\bullet$ & $\bullet$ & $\bullet$ & $\bullet$ & $\bullet$ & $\bullet$ \\
\hline 19 & Rear bumper line & $\bullet$ & $\bullet$ & $\bullet$ & $\bullet$ & $\bullet$ & $\bullet$ & $\bullet$ & $\bullet$ & $\bullet$ & $\bullet$ & $\bullet$ & $\bullet$ \\
\hline
\end{tabular}

2) Components. Common features of vehicles, such as headlights, tires, mirrors, etc., are easily identifiable. Using lines and shadings to express these components can display more information about the shape, texture and other details (Table 2). 
Table 2 Components.

\begin{tabular}{|c|l|l|l|l|l|l|l|l|l|l|l|l|l|}
\hline No. & \multicolumn{1}{|c|}{ Features } & \multicolumn{3}{|c|}{ Single Line } & \multicolumn{3}{c|}{ Line \& Surf. } & \multicolumn{3}{c|}{ Light color } \\
\hline 1 & Roof line & $\bullet$ & $\bullet$ & $\bullet$ & $\bullet$ & $\bullet$ & $\bullet$ & $\bullet$ & $\bullet$ & & $\bullet$ & $\bullet$ & $\bullet$ \\
\hline 2 & $\begin{array}{l}\text { Front windscreen } \\
\text { profile }\end{array}$ & $\bullet$ & $\bullet$ & $\bullet$ & $\bullet$ & $\bullet$ & $\bullet$ & $\bullet$ & $\bullet$ & $\bullet$ & $\bullet$ & $\bullet$ & $\bullet$ \\
\hline 3 & Front hood line & $\bullet$ & $\bullet$ & $\bullet$ & $\bullet$ & $\bullet$ & $\bullet$ & $\bullet$ & $\bullet$ & $\bullet$ & $\bullet$ & $\bullet$ & $\bullet$ \\
\hline 4 & Side hood line & $\bullet$ & $\bullet$ & $\bullet$ & $\bullet$ & $\bullet$ & $\bullet$ & $\bullet$ & $\bullet$ & $\bullet$ & $\bullet$ & $\bullet$ & $\bullet$ \\
\hline 5 & Grille line & $\bullet$ & $\bullet$ & $\bullet$ & $\bullet$ & $\bullet$ & $\bullet$ & $\bullet$ & $\bullet$ & $\bullet$ & $\bullet$ & $\bullet$ & $\bullet$ \\
\hline 6 & Front bumper line & $\bullet$ & $\bullet$ & $\bullet$ & $\bullet$ & $\bullet$ & $\bullet$ & $\bullet$ & $\bullet$ & $\bullet$ & $\bullet$ & $\bullet$ & $\bullet$ \\
\hline 7 & Middle line & $\bullet$ & $\bullet$ & $\bullet$ & $\bullet$ & $\bullet$ & $\bullet$ & $\bullet$ & $\bullet$ & & & & \\
\hline 8 & Side window profiles & $\bullet$ & $\bullet$ & $\bullet$ & $\bullet$ & $\bullet$ & $\bullet$ & $\bullet$ & $\bullet$ & $\bullet$ & $\bullet$ & $\bullet$ & $\bullet$ \\
\hline 9 & Waist line & $\bullet$ & $\bullet$ & $\bullet$ & $\bullet$ & $\bullet$ & $\bullet$ & $\bullet$ & $\bullet$ & $\bullet$ & $\bullet$ & $\bullet$ & $\bullet$ \\
\hline 10 & Shoulder line & $\bullet$ & $\bullet$ & $\bullet$ & $\bullet$ & $\bullet$ & $\bullet$ & & $\bullet$ & & $\bullet$ & $\bullet$ & $\bullet$ \\
\hline 11 & Side scuff line & $\bullet$ & $\bullet$ & & & & & & & & & $\bullet$ & \\
\hline 12 & Skirt line & $\bullet$ & $\bullet$ & $\bullet$ & $\bullet$ & $\bullet$ & $\bullet$ & & $\bullet$ & $\bullet$ & & & \\
\hline 13 & Side chassis line & $\bullet$ & $\bullet$ & $\bullet$ & $\bullet$ & $\bullet$ & $\bullet$ & & $\bullet$ & $\bullet$ & $\bullet$ & $\bullet$ & $\bullet$ \\
\hline 14 & Wheel cover line & $\bullet$ & $\bullet$ & $\bullet$ & $\bullet$ & $\bullet$ & $\bullet$ & $\bullet$ & $\bullet$ & $\bullet$ & $\bullet$ & $\bullet$ & $\bullet$ \\
\hline 15 & Door profile & $\bullet$ & $\bullet$ & $\bullet$ & $\bullet$ & $\bullet$ & $\bullet$ & & $\bullet$ & $\bullet$ & $\bullet$ & $\bullet$ & $\bullet$ \\
\hline 16 & Rear window profile & $\bullet$ & $\bullet$ & $\bullet$ & $\bullet$ & $\bullet$ & $\bullet$ & $\bullet$ & $\bullet$ & $\bullet$ & $\bullet$ & $\bullet$ & $\bullet$ \\
\hline
\end{tabular}

3) Non-form shading. Its role of the so-called non-form shading is not on the description of the grille, windscreen, wheels and other details, but on the description of the area without any shapes, showing the design materials and other common information. The basic role of non-form shading is to create the style and give the area of color, not only for background information, but also for depth characterization in order to make renderings have more 3D effect (Table 3).

Table 3 Non-form shadings.

\begin{tabular}{|c|l|c|c|c|c|c|c|c|c|c|c|c|c|c|}
\hline No. & \multicolumn{3}{|c|}{ Features } & \multicolumn{3}{c|}{ Single Line } & \multicolumn{3}{c|}{ Line \& Surf. } & \multicolumn{3}{c|}{ Light color } \\
\hline 1 & Tire & $\bullet$ & $\bullet$ & $\bullet$ & $\bullet$ & $\bullet$ & $\bullet$ & $\bullet$ & $\bullet$ & $\bullet$ & $\bullet$ & $\bullet$ & $\bullet$ \\
\hline 2 & Ground & $\bullet$ & $\bullet$ & & $\bullet$ & $\bullet$ & $\bullet$ & $\bullet$ & $\bullet$ & $\bullet$ & $\bullet$ & $\bullet$ & $\bullet$ \\
\hline 3 & Color & & & & & & & & $\bullet$ & $\bullet$ & $\bullet$ & $\bullet$ & $\bullet$ \\
\hline 4 & Environment & $\bullet$ & & & & & & & $\bullet$ & & $\bullet$ & $\bullet$ & \\
\hline
\end{tabular}

4) Form Shading. Form shading is applied to curved surface. However, it may appear anywhere. In the actual sketching it is generally used in the expressions of side door, grille, hood, or front windscreen and so on, to give the performance of its functions and some special texture effect. The wheel pattern makes a car look more solid and increases the strong feel. The form shading tends to use a fade effect of expression, which is based on the general impression rather than the precise positioning of surface, and therefore only use the shadow lines instead of its form (Table 4). 
Table 4 Form shadings.

\begin{tabular}{|c|l|c|c|c|c|c|c|c|c|c|c|c|c|}
\hline No. & \multicolumn{2}{|c|}{ Features } & \multicolumn{3}{|c|}{ Single Line } & \multicolumn{3}{c|}{ Line \& Surf. } & \multicolumn{3}{c|}{ Light color } \\
\hline 1 & Windscreen & $\bullet$ & $\bullet$ & $\bullet$ & $\bullet$ & $\bullet$ & $\bullet$ & $\bullet$ & $\bullet$ & & $\bullet$ & $\bullet$ & $\bullet$ \\
\hline 2 & Headlights & $\bullet$ & $\bullet$ & $\bullet$ & $\bullet$ & $\bullet$ & $\bullet$ & $\bullet$ & $\bullet$ & $\bullet$ & $\bullet$ & $\bullet$ & $\bullet$ \\
\hline 3 & Fog lights & & $\bullet$ & & $\bullet$ & $\bullet$ & $\bullet$ & $\bullet$ & $\bullet$ & & & $\bullet$ & $\bullet$ \\
\hline 4 & Front bumper & $\bullet$ & & & $\bullet$ & $\bullet$ & $\bullet$ & $\bullet$ & $\bullet$ & $\bullet$ & $\bullet$ & $\bullet$ & $\bullet$ \\
\hline 5 & Grille & $\bullet$ & $\bullet$ & & & $\bullet$ & $\bullet$ & $\bullet$ & $\bullet$ & $\bullet$ & $\bullet$ & $\bullet$ & $\bullet$ \\
\hline 6 & Side window & $\bullet$ & $\bullet$ & & $\bullet$ & $\bullet$ & $\bullet$ & $\bullet$ & $\bullet$ & & $\bullet$ & $\bullet$ & $\bullet$ \\
\hline 7 & Wheel hub & $\bullet$ & $\bullet$ & $\bullet$ & $\bullet$ & $\bullet$ & $\bullet$ & & $\bullet$ & $\bullet$ & $\bullet$ & $\bullet$ & $\bullet$ \\
\hline 8 & Wheel cover & $\bullet$ & $\bullet$ & & $\bullet$ & $\bullet$ & $\bullet$ & & $\bullet$ & $\bullet$ & $\bullet$ & $\bullet$ & $\bullet$ \\
\hline 9 & Rear windscreen & $\bullet$ & $\bullet$ & $\bullet$ & $\bullet$ & $\bullet$ & $\bullet$ & $\bullet$ & $\bullet$ & & $\bullet$ & $\bullet$ & $\bullet$ \\
\hline 10 & Taillight & $\bullet$ & $\bullet$ & $\bullet$ & $\bullet$ & $\bullet$ & $\bullet$ & $\bullet$ & $\bullet$ & $\bullet$ & $\bullet$ & $\bullet$ & $\bullet$ \\
\hline 11 & Rear bumper & $\bullet$ & & & $\bullet$ & $\bullet$ & $\bullet$ & $\bullet$ & $\bullet$ & $\bullet$ & $\bullet$ & $\bullet$ & $\bullet$ \\
\hline 12 & Exhaust pipe & & $\bullet$ & $\bullet$ & $\bullet$ & $\bullet$ & $\bullet$ & $\bullet$ & & $\bullet$ & & & $\bullet$ \\
\hline 13 & License plate & $\bullet$ & & & & $\bullet$ & $\bullet$ & $\bullet$ & & $\bullet$ & $\bullet$ & $\bullet$ & $\bullet$ \\
\hline
\end{tabular}

\section{Analysis of Sketch Features}

\subsection{Analysis Principle}

The researches about extracting 3D features of autobody from 2D sketches cover a variety of disciplines, such as cognitive psychology, visual identification, aesthetics, computer graphics, sketching behaviour and so on. In the current rapid development of computer vision and cognitive psychology, stylists are still using 2D sketches on paper to communicate with each other. This shows that people can get the 3D feature information from a perspective of $2 \mathrm{D}$ sketch. In this paper, Analytic Hierarchy Process (AHP) [18] has been applied to study the frequency of features used in the various stages of automotive sketches in order to help the further applications in the real time 3D sketching system development.

The basic idea of it is to, at first, classify the goal into different hierarchies or levels; construct a feature matrix based on the classification of samples; and then to determine the weight of each feature level based on the mutual influence among the levels [19]. The steps used for studying the frequency of features in automotive sketches are shown in Figure 6.

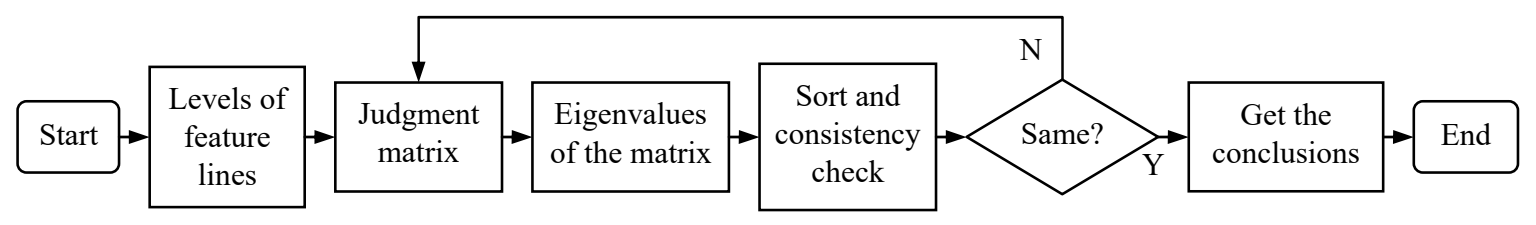

Figure 6 Steps of AHP.

\subsection{Algorithm of AHP Analysis}

The Algorithm can be divided into three steps:

1) Establishing the hierarchy model of features extracted in automotive sketches

Typically, the model contains three levels. The highest level contains only one element, namely, the automotive conceptual sketches. Analyzing the sketches to get the ideal features is known as the goal level. The middle level comprises the features depicting a vehicle, and it may consist of several 
stages, which play an important part in the expression of the vehicle. For example, the threedimensional information is classified into form lines, components, form shading and non-form shading, and they are called the criteria level. The lowest level provides alternative schemes to achieve the features in the sketching, which is also known as the alternatives level [19]. Here three alternatives of sketch are selected for the analysis in the alternatives level, as shown in Figure 7.

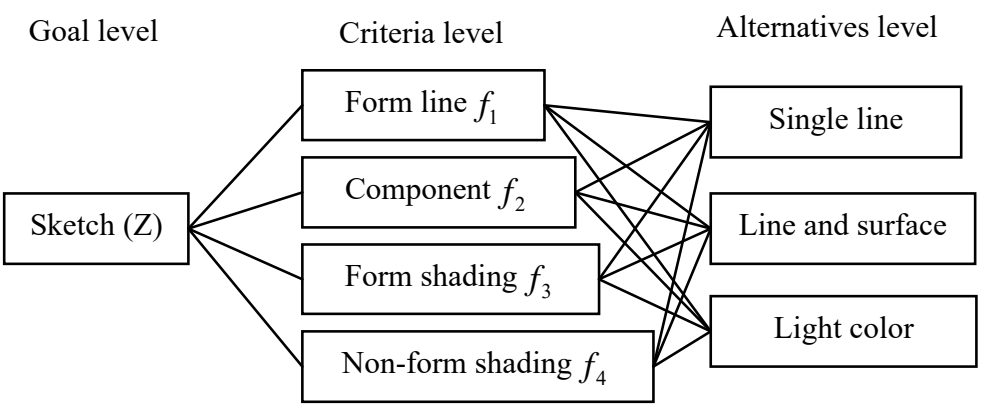

Figure 7 Hierarchical model of automotive features.

2) Constructing paired comparison judgment matrix of features in various levels

The model of automotive features reflects the relationship between the features, but each feature in the criteria level may have a different weight in the evaluation or measurement. According to Saaty theory, the influence factors are used to build the paired comparison judgment matrix. Let the feature set $F=\left\{f_{1}, \cdots, f_{n}\right\}$ containing $\mathrm{n}$ automotive features, which impact on an object $Z$. While analyzing, taking two features $f_{i}$ and $f_{j}$ each time, $s_{i j}$ is used to indicate the influence factor of $f_{i}$ and $f_{j}$ specific to $Z$, and vice versa, $s_{j i}=1 / s_{i j}$ stands for the influence factor of $f_{j}$ and $f_{i}$ specific to $Z$. All the result of the comparison uses the matrix $S=\left(s_{i j}\right)_{n \times n}$, and $S$ is the pairwise comparison judgment matrix between $Z$ and $F$ [20]. At the same time, the number 1 9 and the reciprocal are used as the weight or scale of influence factors, to indicate the degree of their impact on the automotive form, as shown in Table 5. The comparison judgment matrix $S$ can be constructed according to Table 1, and the judgment of $n(n-1) / 2$ times should be conducted in each pair. Here, the comparison judgment is carried on between two adjacent features, it is not that one compares with the rest, i.e. $n-1$ times. This ensures that if there is a judgment which produces errors, the failure does not affect on the reasonable sort of whole features.

Table 5 Scales of influence factor $S_{i j}[13]$.

\begin{tabular}{|c|l|}
\hline Scale & \multicolumn{1}{c|}{ Meaning } \\
\hline 1 & The same importance of both features \\
\hline 3 & The former was slightly important than the latter \\
\hline 5 & The former is more important than the latter obviously \\
\hline 7 & The former is more important than the latter strongly \\
\hline 9 & The former is more important than the latter extremely \\
\hline $2,4,6,8$ & Middle values between any two values above \\
\hline Reciprocal & $\begin{array}{l}\text { If the ratio of the feature importance } i \text { and } j \text { is } S_{i j} \text {, so the one of } j \\
\text { and } i \text { is } S_{i i}=1 / S_{i j} .\end{array}$ \\
\hline
\end{tabular}

3) Feature ordering and consistency checking

The $S$ is corresponding to the eigenvector $W^{T} \equiv\left(\omega_{1}, \cdots, \omega_{2}\right)$ of the maximum eigenvalue $\lambda_{\max }$. After normalized, it is the ordered weight of importance from the factor at the same level to the 
corresponding factor at the previous level. The process is known as the hierarchical single ordering [19].

$$
\lambda_{\max _{i}}=\sum_{j=1}^{n} s_{i j} \frac{\omega_{j}}{\omega_{i}} .
$$

The consistency checking of the judgment matrix is as follows:

i) Calculate the consistency index $\mu$

$$
\mu=\frac{\lambda_{\max }-n}{n-1}
$$

ii) Find the corresponding average random consistency index $R I$.

$$
R I=\frac{\bar{\lambda}_{\max }-n}{n-1}
$$

Where $n=1, \cdots, 9 ; \bar{\lambda}_{\max }$ is the average of the maximum eigenvalue; the matrix of 500 samples is used to obtain RI values by the random method, as shown in Table 6 .

iii) Calculate the ratio of consistency $\mathrm{CR}$.

$$
C R=\frac{\mu}{R I} .
$$

When $C R<0.03$, the consistency of judgment matrix is acceptable, otherwise the judgment matrix should be amended appropriately. The ordered weights for the expression of autobody form in the criteria level can be obtained finally by calculating the weight vectors of feature lines used in the automotive sketches, and then the feature with the highest frequency is found out.

\subsection{Analysis of Form Line}

The hierarchical model of form lines in front of autobody has been constructed as shown in Figure 8.

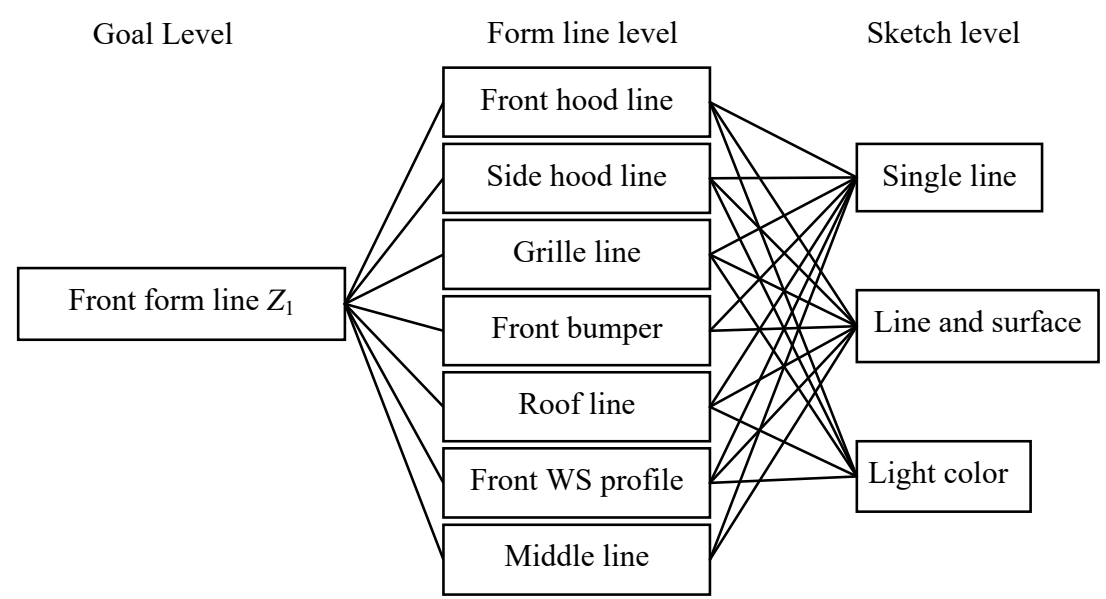

Figure 8 Hierarchical model of the front form lines. 
The form lines are compared in pairs. For instance, the front line of hood and the side edges of the hood are all used in the twelve sketches (as shown in Table 1), they have the same importance, the influence factors are defined as 1; the roof lines are used 11 times, the influence factor of roof line to the front line of hood is $1 / 2$, the one of the front line of hood to the roof line is 2 , reversely. According to the above conclusion, the comparison judgment matrix $S_{1}$ of form lines has been constructed. The maximum eigenvalue of $S_{1}$ is denoted as $\lambda_{\max 1}$.

After calculating the matrix $S_{1}$, the eigenvectors as the weights of the seven form lines are obtained, which is corresponding to the maximum eigenvalue $\lambda_{\max 1}$. The weights are ordered in eigenvector. The most important form line is in the first place, as shown in Table 7.

The similar models, like the one shown in Figure 8, can also be constructed for the form lines in the side and the rear of autobody. Their comparison judgment matrices are $S_{2}$ and $S_{3}$, and their maximum eigenvalues are $\lambda_{\max 2}$ and $\lambda_{\max 3}$. The ordered results are shown in Table 8 and Table 9.

Table 7 Sorting result of the front form lines.

\begin{tabular}{|c|c|c|c|}
\hline Feature & Eigenvector & Weight sorting & \\
\hline Front hood line & 0.8053 & 1 & \multirow{7}{*}{$S_{1}=\left[\begin{array}{ccccccc}1 & 1 & 1 & 1 & 1 & 1 / 2 & 1 / 4 \\
1 & 1 & 1 & 1 & 1 & 1 / 2 & 1 / 4 \\
1 & 1 & 1 & 1 & 1 & 1 / 2 & 1 / 4 \\
1 & 1 & 1 & 1 & 1 & 1 / 2 & 1 / 4 \\
2 & 2 & 2 & 2 & 2 & 1 & 1 / 3 \\
2 & 2 & 2 & 2 & 2 & 1 & 1 / 3 \\
4 & 4 & 4 & 4 & 4 & 3 & 1\end{array}\right]$} \\
\hline Side hood line & 0.8053 & 1 & \\
\hline Grille line & 0.8053 & 1 & \\
\hline $\begin{array}{l}\text { Front bumper } \\
\text { bottom line }\end{array}$ & 0.8053 & 1 & \\
\hline $\begin{array}{l}\text { Front windscreen } \\
\text { profile }\end{array}$ & 0.8053 & 1 & \\
\hline Roof line & 0.3358 & 5 & \\
\hline Middle line & 0.1774 & 7 & \\
\hline
\end{tabular}

Table 8 Sorting result of the side form lines.

\begin{tabular}{|c|c|c|c|c|c|c|c|c|c|c|c|}
\hline Feature & Eigenvector & Weight sorting & \multirow{10}{*}{$S_{2}=$} & \multirow{10}{*}{\multicolumn{2}{|c|}{$\begin{array}{ll}1 & 1 \\
1 & 1 \\
2 & 2\end{array}$}} & & \multirow{3}{*}{$\begin{array}{l}1 / 2 \\
1 / 2\end{array}$} & \multirow{3}{*}{$\begin{array}{l}1 / 2 \\
1 / 2\end{array}$} & \multirow{3}{*}{$\begin{array}{l}1 / 2 \\
1 / 2\end{array}$} & \multirow{3}{*}{\multicolumn{2}{|c|}{$\left.\begin{array}{ll}1 / 2 & 1 / 9 \\
1 / 2 & 1 / 9\end{array}\right]$}} \\
\hline Waist line & 0.9482 & 1 & & & & & & & & & \\
\hline Wheel cover line & 0.9482 & 1 & & & & & & & & & \\
\hline Side window & 0.1344 & 3 & & & 2 & & 1 & 1 & 1 & 1 & $1 / 8$ \\
\hline $\begin{array}{l}\text { protiles } \\
\text { Shoulder line }\end{array}$ & 01344 & & & & 2 & 1 & 1 & 1 & 1 & 1 & $1 / 8$ \\
\hline Shoulder line & 0.1344 & 3 & & & 2 & 1 & 1 & 1 & 1 & 1 & $1 / 8$ \\
\hline Skirt line & 0.1344 & 3 & & & 2 & 1 & 1 & 1 & 1 & 1 & $1 / 8$ \\
\hline Side chassis line & 0.1344 & 3 & & & 2 & 1 & 1 & 1 & 1 & 1 & $1 / 8$ \\
\hline Door profile & 0.1344 & 3 & & & 9 & 8 & 8 & 8 & 8 & 8 & 1 \\
\hline Side scuff line & 0.0729 & 8 & & & & & & & & & \\
\hline
\end{tabular}

Table 9 Sorting result of the rear form lines.

\begin{tabular}{|c|c|c|c|}
\hline Feature & Eigenvector & Weight sorting & \multirow{5}{*}{$=\left[\begin{array}{cccc}1 & 1 & 1 & 1 / 2 \\
1 & 1 & 1 & 1 / 2 \\
1 & 1 & 1 & 1 / 2 \\
2 & 2 & 2 & 1\end{array}\right]$} \\
\hline $\begin{array}{l}\text { Rear window } \\
\text { profile }\end{array}$ & 0.7559 & r 5 & \\
\hline $\begin{array}{l}\text { Front trunk lid } \\
\text { line }\end{array}$ & 0.7559 & 1 & \\
\hline $\begin{array}{l}\text { Side trunk lid } \\
\text { line }\end{array}$ & 0.7559 & 1 & \\
\hline $\begin{array}{l}\text { Rear bumper } \\
\text { line }\end{array}$ & 0.3780 & 4 & \\
\hline
\end{tabular}




\subsection{Analysis of Component}

Similarly, according to Table 2, the comparison judgment matrices of components in the front, side and rear of autobody are $S_{4}, S_{5}$ and $S_{6}$, and their maximum eigenvalues are $\lambda_{\max 4}, \lambda_{\max 5}$ and $\lambda_{\max 6}$, respectively. The ordered results are shown in Table 10 12.

Table 10 Sorting result of the front components.

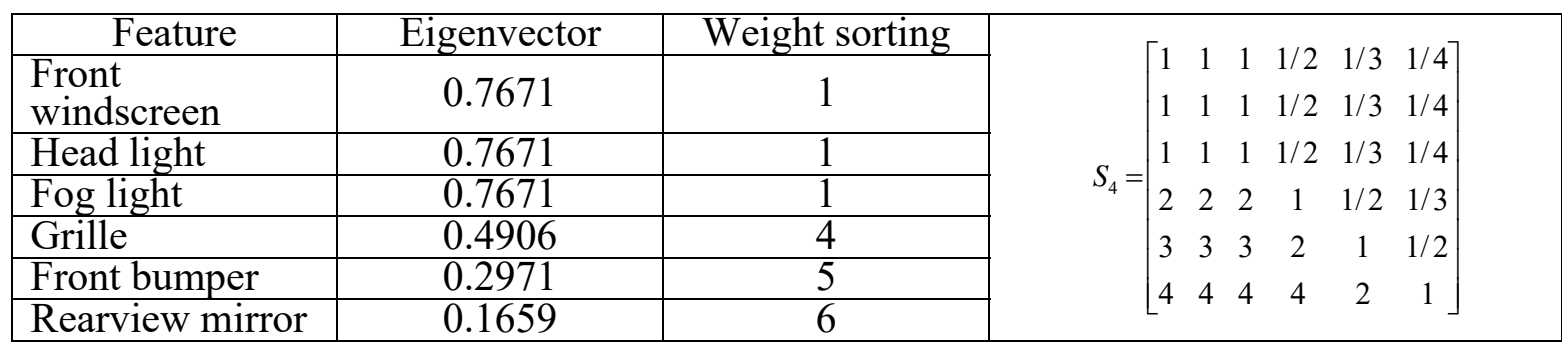

Table 11 Sorting result of the side components.

\begin{tabular}{|c|c|c|c|c|c|c|c|}
\hline Feature & Eigenvector & Weight sorting & \multirow{6}{*}{$S_{5}=$} & \multirow{3}{*}{\multicolumn{4}{|c|}{$\left[\begin{array}{ccccc}1 & 1 / 2 & 1 / 8 & 1 / 8 & 1 / 9 \\
2 & 1 & 1 / 7 & 1 / 7 & 1 / 8\end{array}\right.$}} \\
\hline Wheel & 0.7477 & 1 & & & & & \\
\hline Side window & 0.4639 & 2 & & & & & \\
\hline Side scuff & 0.0841 & 3 & & 8 & 1 & 1 & $1 / 2$ \\
\hline Door handle & 0.0841 & 3 & & 8 & 1 & 1 & $1 / 2$ \\
\hline Side turn light & 0.0589 & 5 & & 9 & 2 & 2 & 1 \\
\hline
\end{tabular}

Table 12 Sorting result of the rear components.

\begin{tabular}{|c|c|c|c|c|c|c|c|c|}
\hline $\begin{array}{c}\text { Feature } \\
\text { Rear humner }\end{array}$ & Eigenvector & Weight sorting & \multirow{5}{*}{\multicolumn{2}{|c|}{$S_{6}=\left[\begin{array}{l}2 \\
2 \\
3\end{array}\right.$}} & \multirow{2}{*}{\multicolumn{4}{|c|}{$\begin{array}{llll}1 & 1 / 2 & 1 / 2 & 1 / 3 \\
1 & 1 / 2 & 1 / 2 & 1 / 3\end{array}$}} \\
\hline $\begin{array}{l}\text { Rear bumper } \\
\text { Taillight }\end{array}$ & $\begin{array}{l}0.7452 \\
0.7452\end{array}$ & $\begin{array}{l}1 \\
1\end{array}$ & & & & & & \\
\hline License plate & 0.4166 & $\frac{1}{3}$ & & & 2 & 1 & 1 & $1 / 2$ \\
\hline Rear windscreen & 0.4166 & 3 & & & 2 & 1 & 1 & $1 / 2$ \\
\hline exhaust pipe & 0.2207 & 5 & & & & 2 & 2 & 1 \\
\hline
\end{tabular}

\subsection{Analysis of Form Shading}

According to Table 4, the comparison judgment matrices of form shadings in the front, side and rear of autobody are $S_{7}, S_{8}$ and $S_{9}$, and their maximum eigenvalues are $\lambda_{\max 7}, \lambda_{\max 8}$ and $\lambda_{\max 9}$, respectively. The ordered results are shown in Table 13 15.

Table 13 Sorting result of the front form shadings.

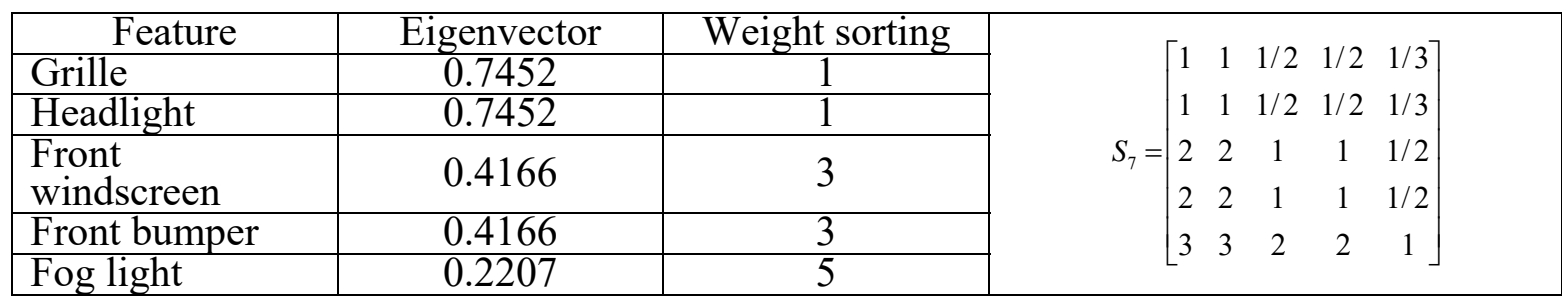


Table 14 Sorting result of the side form shadings.

\begin{tabular}{|c|c|c|c|}
\hline Feature & Eigenvector & Weight sorting & $1 / 2 \quad 1 / 2]$ \\
\hline Wheel hub & 0.6667 & 1 & $\begin{array}{lll}1 / 2 & 1 / 2\end{array}$ \\
\hline Side window & 0.3333 & 3 & $S_{8}=2$ \\
\hline Wheel cover & 0.3333 & 3 & {$[2$} \\
\hline
\end{tabular}

Table 15 Sorting result of the rear form shadings.

\begin{tabular}{|c|c|c|c|c|c|c|c|c|}
\hline Feature & Eigenvector & Weight sorting & \multirow{6}{*}{$S_{9}=$} & \multirow{4}{*}{\multicolumn{5}{|c|}{$\begin{array}{ccccc}1 & 1 / 2 & 1 / 2 & 1 / 4 & 1 / \\
2 & 1 & 1 & 1 / 3 & 1 / \\
2 & 1 & 1 & 1 / 3 & 1 /\end{array}$}} \\
\hline Taillight & 0.6583 & 1 & & & & & & \\
\hline Rear windscreen & 0.2381 & 2 & & & & & & \\
\hline Rear bumper & 0.2381 & 2 & & & & & & $1 / 3$ \\
\hline exhaust pipe & 0.1409 & 4 & & 4 & & 3 & 1 & 1 \\
\hline License plate & 0.1409 & 4 & & 4 & & 3 & 1 & 1 \\
\hline
\end{tabular}

\subsection{Analysis of Non-form Shading}

The comparison judgment matrix $S_{10}$ and its maximum eigenvalue $\lambda_{\max 10}$ are defined according to Table 3 for the non-form shadings. The weights are ordered in eigenvector, and the most important non-form shading is listed on the top of Table 16.

Table 16 Sorting result of the rear form shadings.

\begin{tabular}{|c|c|c|c|c|c|c|c|}
\hline Feature & Eigenvector & Weight sorting & \multirow{5}{*}{$S_{10}=\left[\begin{array}{l}1 \\
2 \\
8 \\
9\end{array}\right.$} & \multirow{3}{*}{\multicolumn{4}{|c|}{$\left[\begin{array}{cccc}1 & 1 / 2 & 1 / 7 & 1 / 5 \\
2 & 1 & 1 / 6 & 1 / 8\end{array}\right.$}} \\
\hline Tire & 0.8273 & 1 & & & & & \\
\hline Ground & 0.5457 & 2 & & & & & \\
\hline Color & 0.1115 & 3 & & 8 & 6 & 1 & \\
\hline Environment & 0.0736 & 4 & & & 7 & 2 & \\
\hline
\end{tabular}

\subsection{Result Analysis}

Through the calculations of above, the average value $\bar{\lambda}_{\max i}$ of the maximum eigenvalue $\lambda_{\max i}$ is obtained, and the consistency index $\mu$ and the ratio of consistency $C R_{i}$ are also calculated out by using the consistency index $R I_{i}$ from Table 6 . When $C R<0.03$, the consistency of judgment matrix is acceptable. The index calculation results are shown in Table 17.

Table 17 Result of consistency calculation.

\begin{tabular}{|c|c|c|c|c|c|c|c|}
\hline Feature & $\lambda_{\max i}$ & $\bar{\lambda}_{\max i}$ & $\mu_{i}$ & $n_{i}$ & $R I_{i}$ & $C R_{i}$ & Result \\
\hline Z1 & 7.0270 & 5.2322 & 0.0045 & 7 & 1.32 & 0.0034 & Ok. \\
\hline Z2 & 8.0525 & 5.2322 & 0.0075 & 8 & 1.41 & 0.0053 & Ok. \\
\hline Z3 & 4.0000 & 5.2322 & 0 & 4 & 0.90 & 0 & Ok. \\
\hline Z4 & 6.0368 & 5.2322 & 0.0074 & 6 & 1.24 & 0.0059 & Ok. \\
\hline Z5 & 5.0907 & 5.2322 & 0.0227 & 5 & 1.12 & 0.0202 & Ok. \\
\hline Z6 & 5.0133 & 5.2322 & 0.0033 & 5 & 1.12 & 0.0029 & Ok. \\
\hline Z7 & 5.0133 & 5.2322 & 0.0033 & 5 & 1.12 & 0.0029 & Ok. \\
\hline Z8 & 3.0000 & 5.2322 & 0 & 3 & 0.58 & 0 & Ok. \\
\hline Z9 & 5.0264 & 5.2322 & 0.0066 & 5 & 1.12 & 0.0059 & Ok. \\
\hline Z10 & 4.0638 & 5.2322 & 0.0213 & 4 & 0.90 & 0.0236 & Ok. \\
\hline
\end{tabular}

By validating, the calculation results of consistency index can disprove that the extraction and classification of various features in the criteria level are correct and reasonable. Therefore, the 
stroke features contained in the forms of single line, line and surface combined and light color can be summarized as follows:

All the form lines contained in the single line form with the higher frequency appeared are those around engine hood, grille, all windows, front and rear bumpers, waist, wheel cover, shoulder, skirt, side chassis, door, trunk lid. Single lines are also appeared in most of the components and some of form shadings, and give priority to the front windscreen, headlights and taillights, wheels, side windows, and shadows as well. Non-form shadings take a very small proportion in the single line form, are generally applied only to the modification of tire and ground.

The form line taking a large proportion in the form of line and surface combined with the higher frequency appeared are those around engine hood, grille, front and rear bumpers, waist, wheel cover, side window, shoulder, rear windscreen and trunk lid. Most of components and form shadings are included in the form of line and surface combined, such as front and rear windscreen, headlights, fog lights, grille, front bumper, wheel, side windows, rear bumper, taillights, and shadows, etc. Non-form shadings also take a very small proportion in the form of line and surface combined, generally used in the tire and ground modification.

Form lines also take a large proportion in the form of light color, and the lines with the higher frequency appeared are those around the front and side of engine hood, grille, front and rear bumpers, waist, wheel covers and trunk lid. Components and form shadings are also appeared in this form, mainly in headlights, fog lights, grille, front bumper, wheel, side windows, rear bumper, license plate, tail lights and shadows. The non-form shading accounts for a large proportion and is also used in the body color and environment, except for the modification of tires and ground.

\section{Conclusions}

Based on the analysis of features used in automotive styling, this paper has investigated three typical automotives sketches, which are in the forms of single line, line and surface combined and light color, by dividing and extracting their features. AHP method has been then applied to analyze and verify the importance of features in the $2 \mathrm{D}$ sketches. The method supplies a solution for analyzing a variety of features, multiple stages and a variety of complex issues based on the weighted qualitative judgments and quantitative calculation, and it is suitable for the study of feature extraction, classification and verification of automotive sketches. The results show that the importance order of features used in most of sketches is reasonable, and meets most of stylists' creative thinking.

The results also demonstrate that the hierarchical models and the judgment matrices work well, the extractions and classifications are reasonable.

The method of feature line classification, extraction experiment to sketches, and AHP verification give a way to solve the problem of feature identification and extraction in 2D sketches, which contain 3D information. This study is not only important significance for the development of three-dimensional real-time sketching system of automobile, but also can be used as a reference in automotive styling.

\section{Acknowledgements}

This work is supported by the Science and Technology Plan Projects of Zhejiang Province under the Grant No. 2013C31085. 


\section{References}

[1] Hu, W. P., Hu, Z. M., et al, Application of digital Virtual Reality Techniques in Industrial Product Model Design [J], Packing Engineering, Vol. 25, No. 2 (2004): 151-153.

[2] Ueng, S. K., Efficient Streamline, Stream Ribbon, and Stream Tube Constructions on Instructions Grids [J], IEEE Transactions on Visualization and Computer Graphics, Vol. 2, No. 2 (1996): 100-108.

[3] Knight, D., Mallinson, G., Visualizing Unstructured Flow Data Using Dual Stream Functions [J], IEEE Transactions on Visualization and Computer Graphics, Vol.2, No. 4 (1996): 355-363.

[4] Nielson, G. M., Tools for Computing Tangent Curves for Linearly Varying Vector Fields over Tetrahedral Domains [J], IEEE Transactions on Visualization and Computer Graphics, Vol. 5, No. 4 (1999): 360-372.

[5] Yang, G., Cheng, S., Tetrahedron-based constructing 3D streamlines in visualization [J], Journal of Beijing University of Aeronautics and Astronautics, Vol. 34, No. 9 (2008): 1061-1064.

[6] Sun, K., Zhang, J. and He, G. P., Research on the DDA Precision Interpolation Algorithm for Continuity of Speed and Acceleration [J]. Advances in Mechanical Engineering, No. 6 (2014): 6-10.

[7] Von Funck, Smoke surfaces: an interactive flow visualization technique inspired by real-world flow experiments [J]. Visualization and Computer Graphics, Vol.14, 6 (2008): 1396-1403.

[8] Li, H. S., Yang, Q., and Chen, Q. M., Constructing Streamlines in 3D CFD Flow Field [J], Journal of Beijing University of Aeronautics and Astronautics, Vol. 29, No. 5 (2003): 434-437.

[9] Jobard, B., Lefer, W., Creating Evenly-Spaced Streamlines of Arbitrary Density [J]. In Proceedings of the Euro Graphics Workshop on Visualization in Scientific Computing, (1997): 4355.

[10] Li, X. C., Feature Extraction of Ocean Field Vortices and Visualization Research [D], Ocean University of China, Qingdao, 2009.

[11] Xu, G., Lu, T. J., Approach to Creation of NURBS Virtual Model Based on IGES [J], Aerospace control, Vol. 25, No. 1 (2007): 62-67.

[12] The initial graphics exchange specification (IGES), GB/T 14213-2008, China standard publishing house, Beijing, 2009. 3.

[13] Werner, A., Skalski, K., Reverse Engineering of Free-form Surfaces [J], Journal of Materials Processing Tech, Vol.76, No.1(1998): 128-132.

[14] Bhandarkar, M. P., Downie, B., Migrating from IGES to STEP: One to One Translation of IGES Drawing to STEP Drafting Data [J]. Computer Graph, Vol.13, No. 13(1994): 339-347.

[15] Hu, K. M., Wang, B., Liu, Y., An Extended Schema and Its Production Rule-based Algorithms for Assembly Data Exchange Using IGES [J]. The International Journal of Advanced Manufacturing Technology, Vol. 58, No. 9 (2012): 1155-1170.

[16] Chen, J. Q., Fan, X. F., Hu, H. F., Numerical simulation of the external flow around a car body [J], Journal of Chongqing University, Natural Science Edition, Vol. 27, No. 10 (2004): 134-137.

[17] Jos, S., Interacting with Smoke and Fire in Real Time [J], Communications of the Association for Computing Machinery, Vol. 37, No. 4 (2000): 76-83.

[18] Liu, M., The Research of Technology in OpenGL-based Real-time Rendering of Big Scale Scene [D], Huazhong University of Science and Technology, Wuhan, 2007.

[19] Zhen, F. F., Research and implementation of key technologies in developing rendering engine [D], Shandong University, 2010.

[20] Zhou, T. M., Wu, Y., Wang, Z. F., Implementation of virtual reality techniques in car styling [J], Automotive Engineering, Vol. 23, No.1 (2001): 18-20. 